\title{
Prise en charge du deuil périnatal
}

\section{Julia Rippstein}

Rédactrice Bulletin des médecins suisses

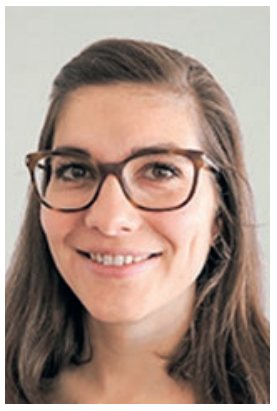

Je n’ai jamais autant côtoyé de médecins, infirmières et autres corps de métier de la santé qu'en 2021. En fait, jusque-là, je n'avais jamais vraiment su ce qu'était être patiente. Je l'ai malheureusement découvert en août dernier, lorsqu'est tombé le verdict d'un décès in utero à la $16^{\mathrm{e}}$ semaine de grossesse. $\mathrm{Si}$, cinq mois plus tard, je peux dire que je me suis remise de cet événement triste et brutal, c'est notamment grâce à toutes les professionnelles de la santé qui m'ont prise en charge et accompagnée durant ces semaines difficiles - j'utilise à dessein la forme féminine, n'ayant vu qu'un homme en tout et pour tout. Parmi toute la documentation que j'ai reçue, l'une souligne d'emblée l'interprofessionnalité: «Nous, médecins de la clinique gynécologique, sommes là pour vous. Nous vous accompagnons et vous soutenons lors de l'adieu à votre enfant, en équipe avec les sagesfemmes et les infirmières.» En gynécologie-obstétrique et en néonatologie, la collaboration interprofessionnelle prend tout son sens. Je m'en suis rendue compte avec cette mésaventure, d'autant plus depuis que je me suis penchée sur le sujet dans le cadre de notre série d'articles sur l'interprofessionnalité.

Durant les 24 heures où j'ai séjourné à l'hôpital pour l'«accouchement silencieux", de l'expression allemande stille Geburt qui décrit l'expulsion d'un fœtus mort in utero, j'ai assisté à un ballet de sagesfemmes: ce sont elles qui se sont occupées de moi le plus étroitement et ont fait en sorte que l'expérience soit la moins douloureuse physiquement et psychiquement pour moi et mon partenaire, présent tout du long. Ce sont elles qui ont servi de fil rouge dans le suivi et étaient aux petits soins de A à Z. Mon compagnon et moi avons également eu un entretien avec une aumônière, qui voulait savoir comment nous voulions dire «au revoir» à notre enfant. Je ne m’attendais pas à être confrontée au service d'aumônerie, mais discuter d'un thème aussi sensible que la mort avec une spécialiste s'est révélé rassurant et bienfaiteur. De nombreuses questions surgissent: Qu'advient-il du corps une fois expulsé? Quelles sont les options de cérémonie? Ces interrogations ont longtemps été négligées lors de la prise en charge de femmes ayant subi une fausse couche. Un accompagnement spirituel, même si ce n'est qu'un bref entretien, est judicieux. Car dire au revoir, par le biais d'un rite, qui peut être le simple fait de se recueillir quelques instants près du bébé, aide à conscientiser la mort et à faire le deuil.
Cette expérience hospitalière m'a montré à quel point le processus était bien rôdé et les rôles clairement définis: chacune sait parfaitement ce qu'elle doit faire et quand, quelles informations transmettre aux collègues à quel moment. Le corps médical n'est intervenu que lorsqu'il fallait effectuer un geste technique pour retirer le placenta. La cheffe de clinique a réalisé l'ultrason pour vérifier qu'aucun reste ne se nichait encore dans l'utérus. Elle a aussi fait le contrôle de suivi plusieurs semaines plus tard. Je n'ai jamais eu l'impression que des informations étaient restées en rade. Signe que l'interprofessionnalité dans ce domaine est éprouvée mais aussi que, hélas, mon cas n'est de loin pas isolé.

Si nous l'avions souhaité, nous aurions pu recevoir un soutien psychologique plus poussé. «Ce que vous vivez est difficile, n'hésitez surtout pas à demander de l'aide à des spécialistes si vous sentez que cela ne va pas, que ce soit maintenant ou plus tard», m'ont répété les médecins. Heureusement, nous n'avons pas eu besoin de recourir à cette aide supplémentaire.

Signe que l'interprofessionnalité dans ce domaine est éprouvée mais aussi que, hélas, mon cas n'est de loin pas isolé.

Ce qui m'amène à conclure: si cette prise en charge interprofessionnelle a largement contribué à ma «guérison", c'est le fait d'en parler autour de moi, avec mon partenaire, ma famille et mes amis proches, qui m'a probablement le plus aidé à accepter cette fausse couche. Revenir sur cette épreuve était certes douloureux, mais nécessaire pour l'assimiler dans un premier temps et passer par-dessus dans un second.

J'aimerais encourager toutes les femmes et tous les couples qui ont traversé la même chose à mettre des mots sur cet événement, à l'extérioriser. Pourtant fréquent, le deuil périnatal reste tabou. Selon l'OFs, le taux de mortalité périnatale en Suisse était de 6,3 cas pour 1000 naissances en 2019 [1]. Les fausses couches spontanées jusqu'à la $20^{\mathrm{e}}$ semaine représentent 15 à $20 \%$ des grossesses confirmées [2], ce qui en fait le problème médical gestationnel le plus répandu. C'est en brisant ce tabou que l'on aide les personnes touchées à surmonter un tel événement.

\section{Références}

1 https://www.bfs.admin.ch/asset/fr/je-f-14.03.04.02.01

2 https://www.agapa-suisseromande.ch/wp/wp-content/ uploads/2017/01/fiche-fausse-couche.pdf 